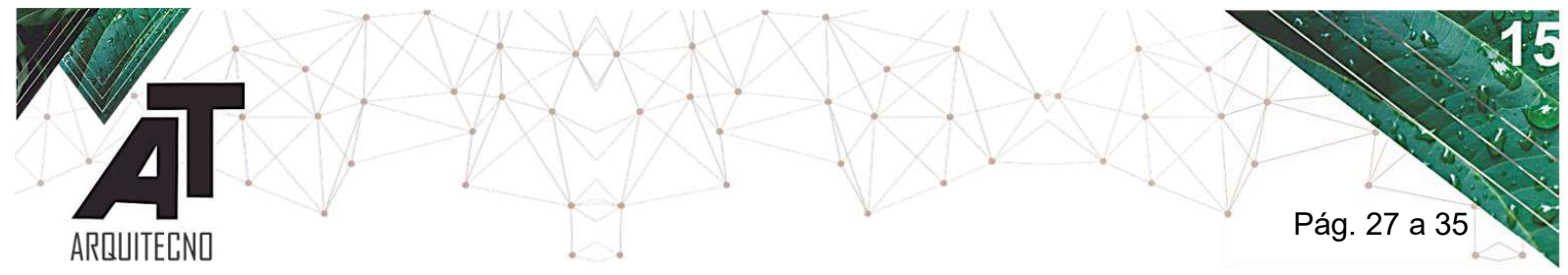

\title{
Análisis de estructuras de Grandes Luces. Estudio de su forma y componentes de diseño, mediante uso de modelos digitales y lectura de la imagen
}

\section{Analysis of structures of great lights. Study of its form and design components, through use of digital models and reading of the image}

Víctor Hugo Cabrera, Daniel Edgardo Vedoya

ITDAHu (Instituto de Investigaciones Tecnológicas para el Diseño Ambiental del Hábitat Humano), Facultad de Arquitectura y Urbanismo, Universidad Nacional del Nordeste, Resistencia, Argentina

vcab.ar18@hotmail.com; devedoya@gmail.com

\section{RESUMEN}

Las tensoestructuras son una tipología de creciente aplicación, cuyo vertiginoso desarrollo se vincula a factores como la producción de piezas industriales especiales y la progresiva aplicación de los principios de las "tensegrity", destacándose su reiterado aprovechamiento en la construcción de grandes instalaciones deportivas. Éstas presentan exigencias técnicas que aquellos sistemas estructurales satisfacen plenamente; para lo cual es relevante contemplar su comportamiento mecánico, estrechamente ligado a la geometría espacial, donde el diseño de sus componentes implica simultáneamente una consideración formal y estructural. No obstante, aún existen ítems poco indagados como los detalles constructivos que, a pesar de constituir un factor clave para el proceso de diseño, y no abundan las soluciones tecnológicas como lo tienen otros sistemas.

El presente trabajo se propone, a partir de un caso estructural, analizar la relación entre la "forma" y las especificaciones técnicas necesarias para su correcta materialización, identificando los criterios de diseño utilizados según los requerimientos a satisfacer.

Desde este enfoque se asocian las soluciones proyectuales a nivel de composición general con los detalles resultantes, apoyándose en métodos de análisis expeditivos y el uso de Modelos Analógicos/Digitales, desplegando interpretaciones sobre la estructura que integran y el conocimiento técnico, como recurso válido de evaluación y diseño.

\begin{abstract}
Tenstructures are a typology of increasing application, whose dizzying development is linked to factors such as the production of special industrial parts and the progressive application of the principles of "tensegrity", highlighting its repeated use in the construction of large sports facilities. These present technical requirements that those structural systems fully satisfy; for which it is relevant to contemplate its mechanical behavior, closely linked to spatial geometry, where the design of its components simultaneously implies a formal and structural consideration. However, there are still little investigated items such as the construction details that, despite being a key factor for the design process, and technological solutions are not abundant as other systems have.

The present work sets out, from a structural case, to analyze the relationship between the "shape" and the technical specifications necessary for its correct materialization, identifying the design criteria used according to the requirements to be satisfied. From this approach, project solutions at the general composition level are associated with the resulting details, relying on expeditious analysis methods and the use of Analog / Digital Models, deploying interpretations about the structure they integrate and technical knowledge, as a valid resource for evaluation and design.
\end{abstract}

PALABRAS CLAVES: Componentes - Detalles - Estadios - Forma Estructural - Tensoestructuras

KEYWORDS: Components - Details - Stadiums - Structural Form - Tenstructures

FECHA DE RECEPCIÓN: 25/05/2020 | FECHA DE ACEPTACIÓN: 30/05/2020

DOI: http://dx.doi.org/10.30972/arq.0154384 


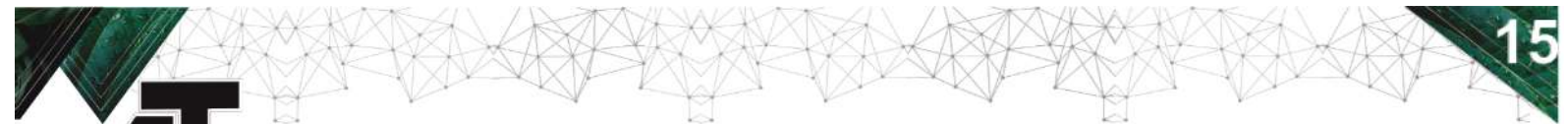

Análisis de estructuras de Grandes Luces. Estudio de su forma y componentes de diseño, mediante uso de modelos digitales y lectura de la imagen

Cabrera, Vedoya

\section{INTRODUCCIÓN}

En el presente resumen, se exponen parte de las tareas de investigación que se inscriben dentro del trabajo sobre el estudio y análisis del comportamiento de estructuras de Grandes Luces de Tracción Pura mediante modelos analógicos y la lectura de la imagen, que toma como caso de estudio al estadio "Wanda Metropolitano" (Madrid, España), el cual posee una cubierta que se incluye dentro de la tipología de las estructuras tensadas.

Uno de los rasgos más significativos de este tipo de resolución estructural es la capacidad de articularse dinámicamente según la disposición de sus componentes en una estrecha relación entre forma y función estática. A partir de ello, se considera como pertinente el empleo de métodos innovadores en el estudio de estructuras, que permitan comprender el comportamiento estructural básico de un sistema de manera expeditiva, como el aplicado en este trabajo, denominado "lectura de la imagen".

Al ver o concebir un edificio con una estructura de grandes luces, lo primero que se presentara ante nuestra visión es un boceto de los rasgos destacables que conforman y definen su geometría esencial, esta responde al contexto de la obra, así como también al tipo de proyecto en lo que se podría definir como sus aspectos "arquitectónicos"; sin embargo, dicha cuestión morfológica tiene una correspondencia directa con una dimensión intrínseca al diseño aunque muchas veces segmentada en su consideración: la tecnológica-estructural. La coherente y armónica relación entre ambos quedara definida según los principios que rigen a los sistemas estructurales naturales y que las tensoestructuras aspiran reproducir.

Por otra parte, las exigencias del mundo actual demandan no sólo la construcción de superficies que cubran grandes espacios sino también que sean eficientes; consideraciones a tener en cuenta en el diseño. Esto se ve reflejado en materia de equipamientos deportivos, donde se han visto asociados el uso de ciertas tipologías estructurales con la función deportiva, especificada en los "estadios".

Es así que, tomando como objeto de estudio la estructura tensada de la cubierta del estadio Metropolitano de Madrid, se plantea realizar un breve recorrido por sus atributos más importantes a fin de reconocer los criterios de diseño empleados, con la intención de revelar la forma en que las decisiones proyectuales determinan las soluciones tecnológicas, en un constante juego entre forma y comportamiento estructural.

De esta manera, el trabajo busca analizar la articulación tecnológica entre sus componentes estructurales, centrando la atención en los detalles resultantes, como medio de reconocer la idoneidad del proceso proyectual, considerando no solo sus componentes físicos, sino también los requerimientos propios del proyecto.

En este sentido, la investigación sobre estructuras es una herramienta que promueva la formación de una visión compleja, poniendo énfasis no solo en potenciar el desarrollo de rasgos técnicos sino incorporándolos a todo proyecto como objetivos de sustentabilidad.

\section{METODOLOGÍA}

La idea de realizar un repaso por los principales componentes o partes de la estructura de cubierta estudiada, vinculándolo a las resoluciones técnicas, parte de exhibir la aplicación, en las diferentes fases del proceso de diseño, de un mismo criterio, donde se integran ideología estructural con los requerimientos propios de cada proyecto. 
Análisis de estructuras de Grandes Luces. Estudio de su forma y componentes de diseño, mediante uso de modelos digitales y lectura de la imagen

Cabrera, Vedoya

\section{DESARROLLO}

\section{Antecedentes/ programa}

Caso Estudiado: "Estadio Metropolitano", sede del Club Atlético de Madrid.

El Estadio Metropolitano, es sede del Club Atlético de Madrid desde el año 2017, construido originalmente para albergar Atletismo en 1997, el edificio original se realizó en base a un diseño propuesto por los arquitectos Cruz y Ortiz, misma firma a cargo de la remodelación.

El viejo diseño, de forma ovalada era conocido por su apodo Estadio de "La Peineta", debido a la similitud de la gradería con el tradicional peine español (Fig. 1).

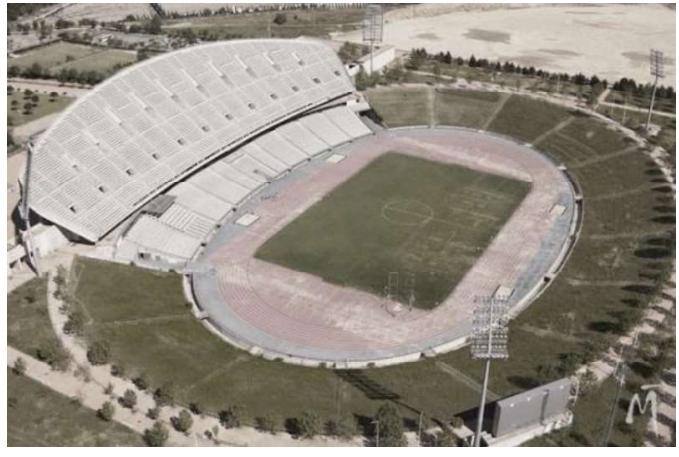

Figura 1: Perspectiva aérea del Antiguo estadio "la Peineta", construcción inaugurada en 1994. Fuente: pgiengineering.com.

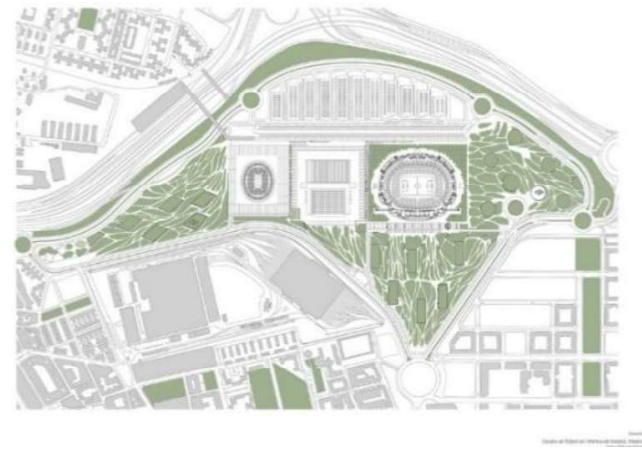

Figura 2: Emplazamiento en Parque Olímpico de la Comunidad de Madrid. Fuente: nuevoestadioatleti.blogspot.com

Ubicado en el barrio de Rosas, integra el sector Oeste del Parque Olímpico de Madrid (Fig. 2); la remodelación se proyectó con una serie de nuevas condiciones muy específicas, para conseguir que una realización de su escala pueda ser autosuficiente en su explotación deportiva, planteando una solución de una potente flexibilidad. Hoy en día es uno de los estadios más modernos a nivel mundial. Entre sus principales premisas pueden citarse:

- Requisitos de Programa: Alto desempeño funcional y alta tecnología.

- Requisitos visuales: Conservar la imagen reconocible del anterior graderío, que la ampliación formara un conjunto armonioso con el antiguo proyecto, integrando ambas "partes", para así lograr representar la nueva imagen del Club. La idea esencial se exhibe en la Fig. 3.

- Requisitos Tecnológicos: Cubierta de grandes luces con forma dinámica, que se adapte a diferentes alturas (Tribuna existente). Asegurar el Confort térmico y niveles de iluminación natural.

Justamente un componente importante en su nueva imagen y conformación es la cubierta, la cual fue concebida en base al diseño estructural del asociado al proyecto, el Ing. Mike Schlaich. El cual Recibió numerosos premios y realizo obras vinculadas al uso de las tensegridades y modalidades de tracción a través de tensores.

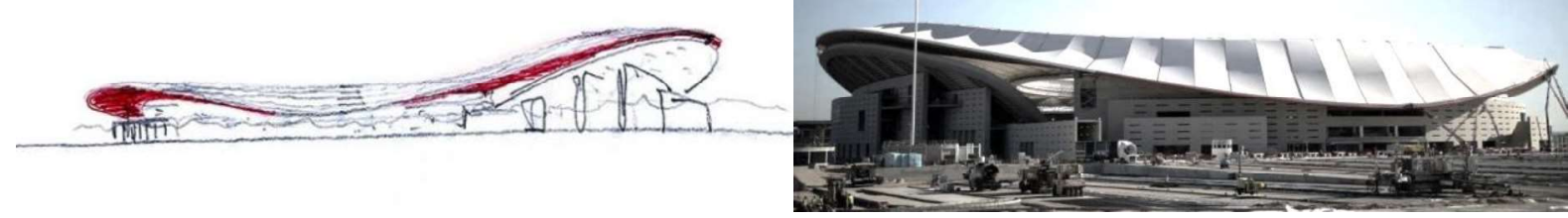

Figura 3: Boceto de Síntesis mental del estadio. Fuente: cruzyortiz.com/portfolio. 
Análisis de estructuras de Grandes Luces. Estudio de su forma y componentes de diseño, mediante uso de modelos digitales y lectura de la imagen

Cabrera, Vedoya

De esta manera, en la imagen del estadio se reconoce por su perfil horizontal, que se integra al paisaje del parque, sobresaliendo sus atributos formales: la composición alargada, los volúmenes articulados del basamento, de imagen moderna, y sus dos partes principales: La tribuna en forma de "Peineta" y la Cubierta sobre el graderío con una silueta dinámica, a modo de "gran manto". Tal como se ilustra en la Fig. 4.

Entre los aspectos que hacen a su categorización, se destaca es el aumento de la capacidad de espectadores, lo cual marca su nivel de elite, pasando de los 20.000 espectadores originales a las 67.703 personas actuales. Tal como se ilustro en la tabla 1 , dar cobijo efectivo ese volumen de personas requiere de $83.000 \mathrm{~m} 2$, asegurando al mismo tiempo, el asoleamiento del campo de juego.

\section{Idea general/ anteproyecto.}

\section{Ampliación sintetizada}

La base del proceso de diseño fue ofrecer una experiencia optimizada para el público, con la creación de un ambiente atractivo y funcional, haciendo hincapié en resaltar los elementos simbólicos del club, piezas arquitectónicas que reflejen su historia, pero al mismo tiempo combinándolo con los elementos nuevos. A partir de ello se derivan las premisas que dan forma a los elementos destacables de la imagen.

Partiendo de su modelo original, la ampliación del estadio consistió en completar el estadio existente en las zonas desocupadas, mediante la construcción de nuevos graderíos continuos (Fig. 5). El conjunto de la estructura tras la ampliación se divide en tres zonas perfectamente diferenciadas, con la cubierta como componente de cierre formal, funcional y estructural (Fig.6):

1. Gran Tribuna principal: Construcción original, a efectos del análisis se denominará "Tribuna".

2. Graderío. Totalidad de las instalaciones del resto del anillo, propios de la nueva construcción. La cubierta ligera, que protege a los espectadores, y se incluye dentro de la tipología de las estructuras tensadas.

3. Cubierta Tensada:

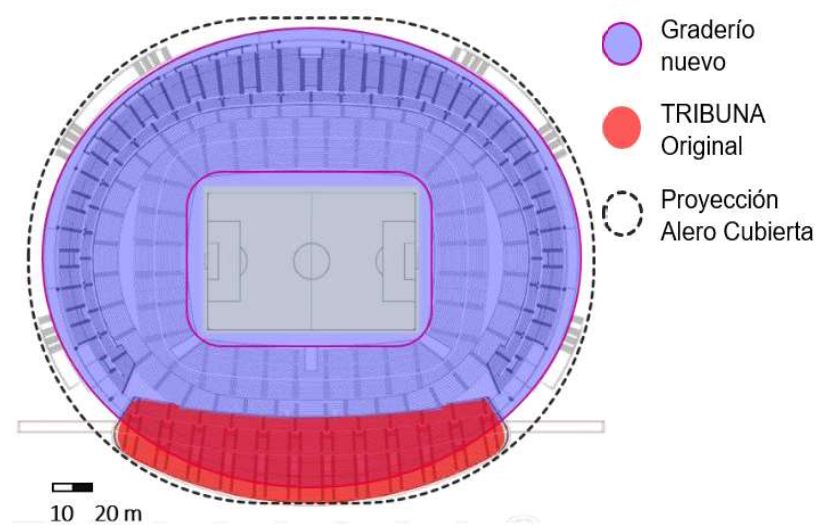

Figura 5: Esquema en planta ampliación de estadio. Fuente: Elaboración Propia en base a Planos Oficiales, encontrados en: nuevoestadioatleti.blogspot.com.

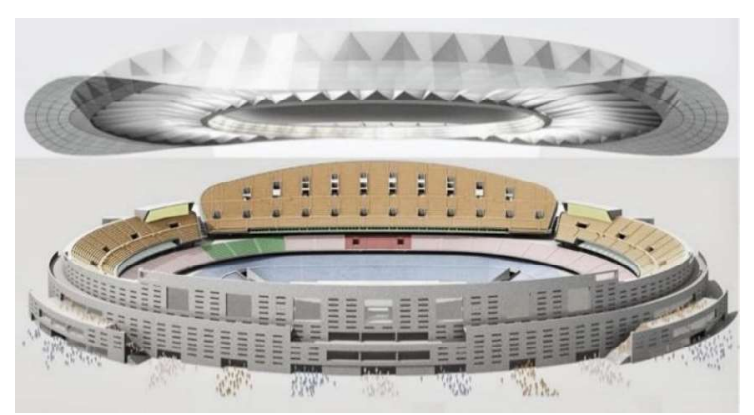

Figura 6: Volumetría estadio. Despiece Componentes Constructivos y espaciales. Fuente: www.cruzyortiz.com

En resumen, el edificio presenta dos lógicas constructivas que se integran en una misma lectura: Lo macizo y pesado del Hormigón, y la ligereza sinuosa de la Cubierta tensada.

Si bien el presente trabajo se enfoca en la estructura de la cubierta se señala de manera precisa, algunos de los aspectos básicos de la estructura general del Estadio.

Para tener una visión global del comportamiento tecnológico, es apropiado señalar que el esquema estructural de los graderíos persigue la misma lógica que la Tribuna principal, dando lugar a una total 


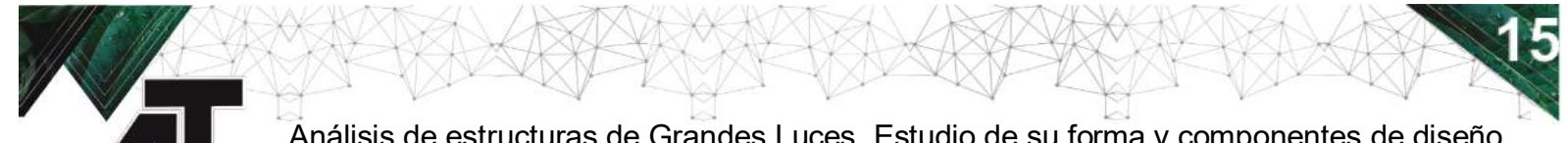

Análisis de estructuras de Grandes Luces. Estudio de su forma y componentes de diseño, mediante uso de modelos digitales y lectura de la imagen

Cabrera, Vedoya

homogeneidad y armonía del conjunto, no solo por la articulación entre sino también por la trama de apoyos generada para la cubierta. La continuidad de la modulación estructural en el espacio favorece la imagen de obra única. Estructuras de hormigón ilustrados en la Fig. 7.
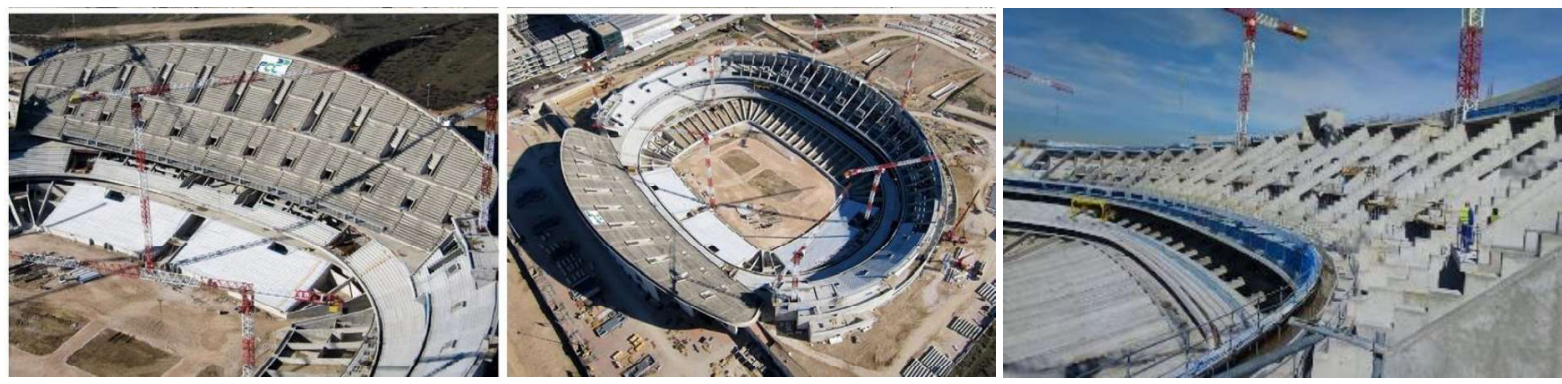

Figura 7: Ampliación de Estructura de Hormigón. Fuente: nuevoestadioatleti.blogspot.com.

El edificio se planteó como un complejo sistema de galerías, dicha tipología estructural, presenta pautas que intervienen en el diseño y trabajo de la cubierta, sintetizados a continuación:

- Los elementos portantes en general son de hormigón armado, con uniones estructurales en diseño de carácter industrial, favoreciendo la homogeneidad formal en las articulaciones.

- Sistemas radiales aporticados, son la base estructural y formal del sistema portante. Parejas de pórticos, cada 4,20 m entre ejes, son los elementos directores de la configuración radial.

- Puntos con relación directa: Los potentes soportes de hormigón de sección pseudo-elíptica que forman el apoyo de la cubierta. Desarrollados a través de tragaderos de luz. (Fig. 8).

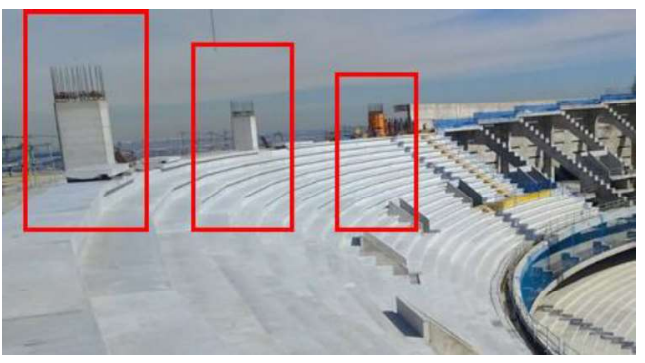

Figura 8: Detalle soportes elevados por sobre el graderío para recibir la cubierta. Fuente: nuevoestadioatleti.blogspot.com

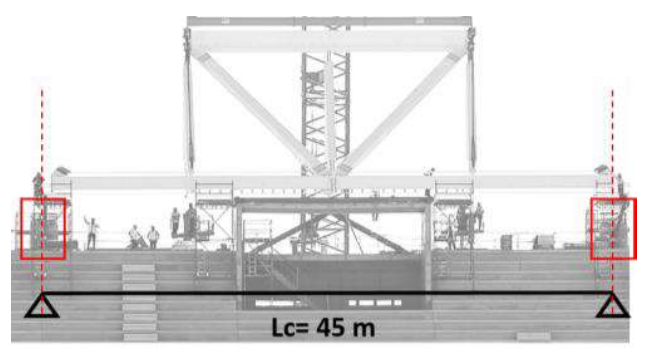

Figura 9: Luces de apoyo de Cerchas. Fuente: Elaboración propia en base a imagen encontrada en: atleticodemadrid.com/galerías

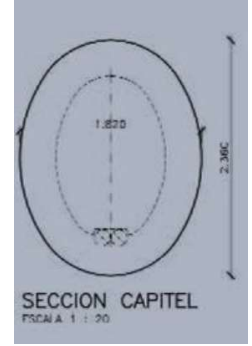

Figura 10: Detalle Capitel. Fuente: Martínez, Gómez N y Schlaich (2009).

Se sitúan 19 soportes, separados entre 40 y 45 m, distribuidos aproximadamente 18 en la zona del nuevo Graderío, y 2 en la Tribuna. La mencionada trama, representa el modulo formal del anillo de compresión, determinando a su vez, nada más y nada menos que sus luces de apoyo. (Fig. 9). Presentan un ensanchamiento a modo de capitel para mejor recepción de cargas (Fig. 10).

A modo de resumen: El conjunto, a partir de una planta ovoidal, organiza los componentes agregados a la tribuna original de manera radial, englobados bajo una misma cubierta, la cual ofrece sombra sobre a las gradas y luz natural campo de futbol.

\section{Cubierta}

La Cubierta ligera expresa el cumplimiento de dos premisas, por un lado, aparece como componente protagonista al desarrollarse elásticamente por todo el estadio, y también por lograr "dialogar" espacialmente con el cuerpo fundacional del proyecto: la "Peineta" (tribuna histórica y volumen con más altura), ya que modifica su altura para cobijarla y "extenderse" por sobre parte de los cerramientos verticales (Figuras 11 y 12 ). 
Análisis de estructuras de Grandes Luces. Estudio de su forma y componentes de diseño, mediante uso de modelos digitales y lectura de la imagen

Cabrera, Vedoya

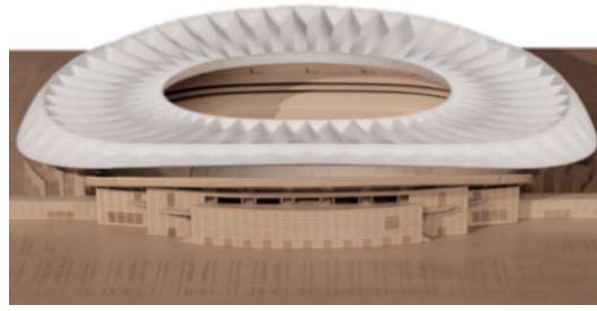

Figura 11: Maqueta del Estadio. Morfología y volumen de la Composición. Fuente: cruzyortiz.com/portfolio.

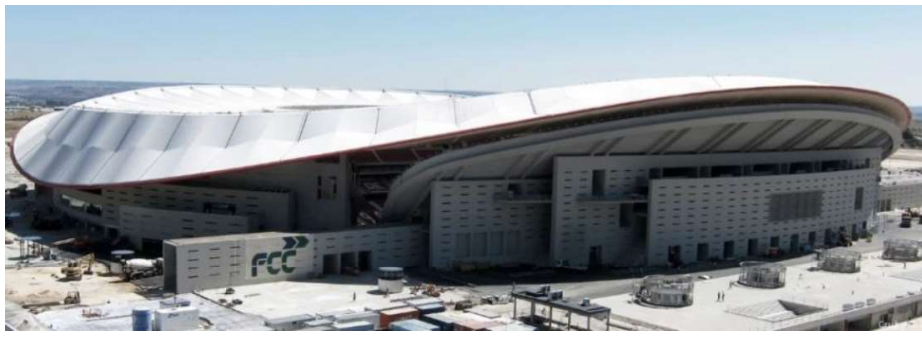

Figura 12: Perspectiva área. Vista a tribuna y perfil de Cubierta. Fuente: mc2.es/proyecto/estadio-metropolitano/

La cubierta es una "estructura en anillo de cables" que está compuesta por un anillo de compresión exterior de acero, un anillo de tracción de cables y dos grupos de cables radiales, que conectan y tensionan a los dos primeros elementos. Esta estructura primaria será recubierta por un material de membrana. El sistema adoptado, presenta desde lo conceptual, referencias a los estudios sobre la "rueda de radios", realizados por Fuller (1975). La cual es una analogía sobre los conceptos fundamentales de la Tensegridad; Fuller recurría a esta comparación muy a menudo e inauguró una nueva era de pensamiento en términos tensionales: compresión discontinua y tracción como sustento principal. Este principio, parece observarse en el diagrama del diseño de la cubierta del estadio.

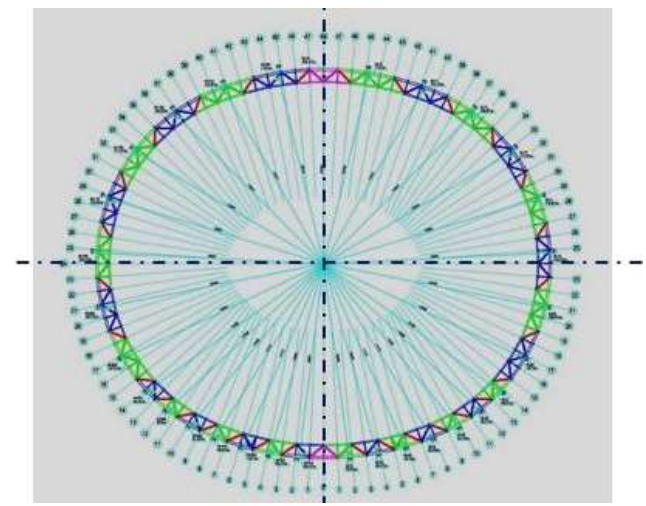

Figura 13: Diagrama estructural. Esquema radial. Fuente: Elaboración propia en base a imagen encontrada en: nuevoestadioatleti.blogspot.com

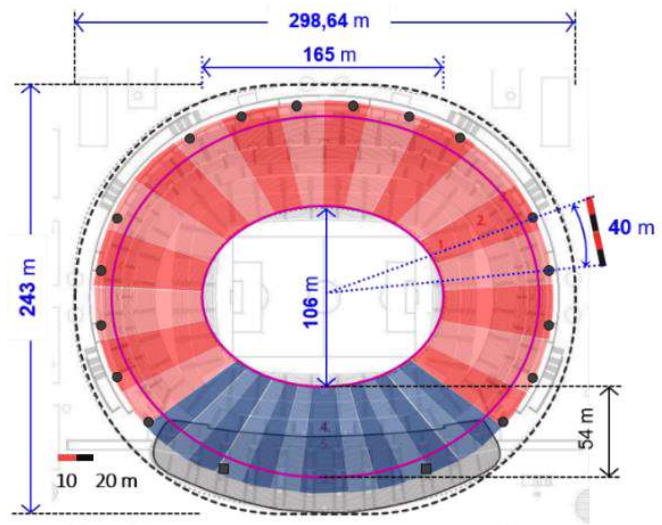

Figura 14: Planta. Modulación y medidas generales. Fuente: Elaboración Propia en base a planos oficiales encontrados en: nuevoestadioatleti.blogspot.com- cruzyortiz.com

Como se ha mencionado, la elección del esquema radial (Fig. 13) determina una tipología estructural, así como también un patrón formal de composición. A partir de su "radios" generadores, se generó la modulación general de la cubierta, tal como se ilustra en la Fig. 14.

Vemos que, en el plano, la estructura es simétrica con respecto a dos ejes (excepto para los apoyos) y su forma se define por secciones concéntricas de círculos; donde una de sus franjas marca el ancho de cubierta (distancia entre los anillos de compresión y tracción), de 54 m.

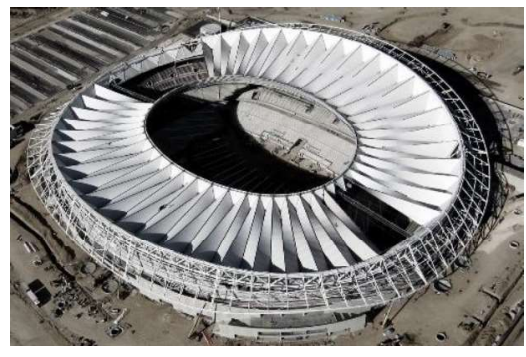

Figura 15: Perspectiva Anillos de Cubierta. Fuente:archdaily.com//time-lapse

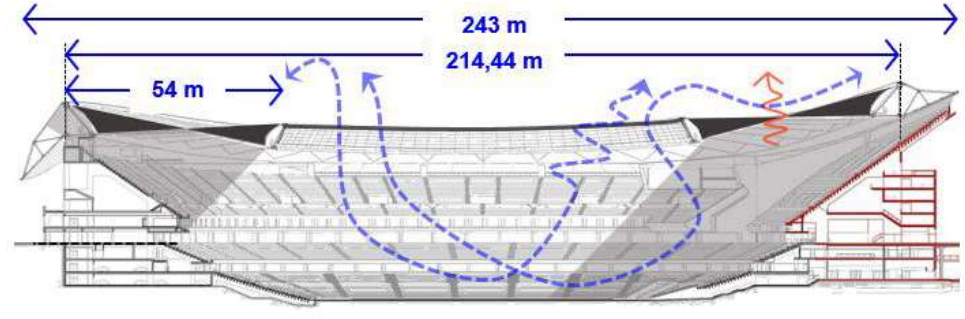

Figura 16: Corte de la Cubierta del Estadio. Fuente: Elaboración propia en base a secciones oficiales encontradas en: cruzyortiz.com 
Análisis de estructuras de Grandes Luces. Estudio de su forma y componentes de diseño, mediante uso de modelos digitales y lectura de la imagen

Cabrera, Vedoya

Los esfuerzos internos determinan la materialidad de los elementos resistentes: Por un lado, la celosía tridimensional de 54 segmentos en acero, trabaja a comprensión mediante perfiles rígidos (Fig. 19), mientras que tensores, en cables de acero, traccionan a todo el sistema (Fig. 20).

Los 108 cables se fijan mediante cabezales industriales, a chapas adosadas en las cerchas y canales abulonados en las bielas flotantes. (Fig. 20). La pendiente de la biela obedece a los ángulos de los tensores, inclinándose para optimizar el trabajo vectorial, actuando como brazo vinculante de las franjas traccionadas -cables- como reacción al empuje (Fig. 21), mediante un sistema tridimensional. Este se rigidiza a través de una elevada tracción y una fabricación precisa.

Por último, las condiciones de vínculo expresan la idoneidad entre tecnología y trabajo mecánico.
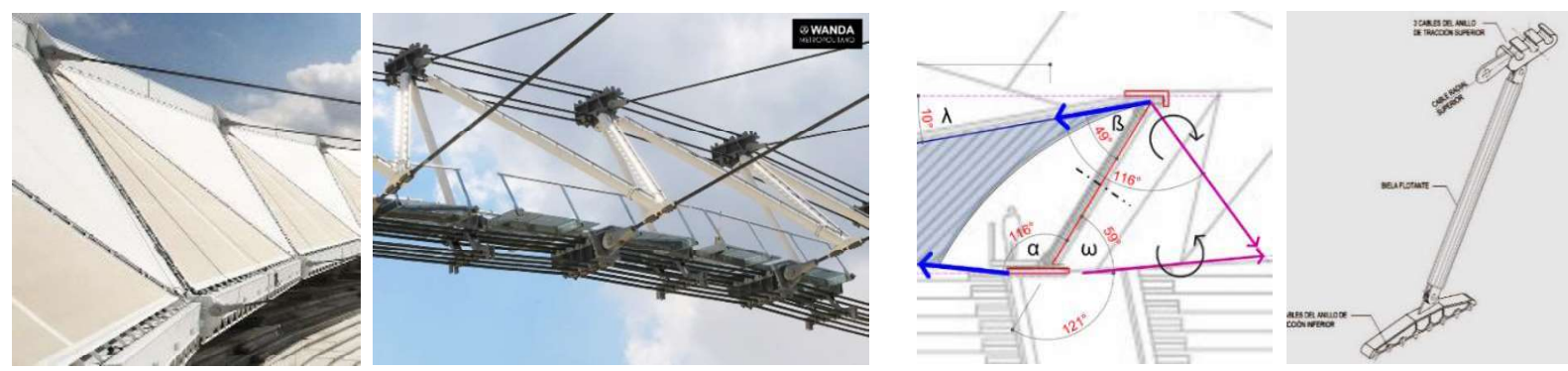

Fig. 20: Anclajes de tensores en celosía y biela (cabezal industrial). Fuente: atleticodemadrid.com/galerías

Fig. 21: Articulaciones. Materialización anillo de tracción. Fuente: Elaboración propia en base a nuevoestadioatleti.blogspot.com y Martínez, Gómez N. y Schlaich (2009).

\section{CONCLUSIONES}

Dentro del ámbito proyectual se ha establecido un par conceptual, definido por la reiterada vinculación entre tipología tensegrity y los estadios deportivos, presente ante la necesidad de cubrir grandes luces. La cubierta doto de unidad a la intervención, con una geometria de diseño que supero las condicionantes tecnicas con acertada resolucion formal. En relacion a ello, el uso del paraboloide hiperbólico como elemento modular, muestra la aplicación de superficies de doble curvatura, en contribución a la capacidad estructural, alto valor estético y reducción de material.

Lo novedoso vendría dado por aplicar esa misma jerarquía de criterio en obras de diversa escala y funcionalidad. Con vistas en poder reproducir la idoneidad de resoluciones técnicas, adaptadas a otros contextos, pero con igual éxito, toma relevancia el manejo de la temática por parte de los profesionales vinculados a la construcción del hábitat. Para contemplar las posibilidades de la tecnología actual y potenciar un diseño eficiente, es indispensable establecer un correcto marco lógico proyectual, lo cual forma parte de las tareas de investigación y favorece la formación de una ideología estructural por parte del proyectista. Esto le permite incorporar una visión más integral sobre la obra, de manera de poder conciliar los requerimientos funcionales y técnicos bajo una misma idea.

Como lo afirman los autores del proyecto, Cruz y Ortiz (2011): "En arquitectura, la hoja nunca está en blanco, siempre hay un lugar, un programa, un contexto". Es así que, de antemano, siempre hay unas condiciones esenciales a las que responder que preceden a la idea y, por ende, a la forma. Entender que el trazo de una geometría responde no solo a esas cuestiones sino también a una forma de comportamiento mecánico es algo que en el ejercicio no siempre goza de consideración, siempre vinculado a un sistema cultural. Por ello, si bien la continuidad en el desarrollo de tensoestructuras tiende al desarrollo de sistemas portantes que, sustentados en conocimientos científicos y movilizados por ejecuciones creativas, habiliten maximizar resultados, el diseño concebido debe poder colocar la potencialidad de dicha tipología al servicio del proyecto en todos sus alcances. 
Análisis de estructuras de Grandes Luces. Estudio de su forma y componentes de diseño mediante uso de modelos digitales y lectura de la imagen

Cabrera, Vedoya

\section{BIBLIOGRAFÍA}

Gómez Jáuregui, V. (2013). Tensegridad, estructuras de compresión flotante. Santander (España). Martínez Calzón, J., Gómez Navarro, M. y Schlaich, M. (2009). El proyecto de la estructura del Estadio Olímpico de Madrid. Madrid (España): Revista de Obras Públicas. № 3.502.

Llorens Duran, José I. (2011). Los detalles constructivos de las tenso estructuras. Barcelona (España): Escuela de Arquitectura.

Revista Expresión Grafica Arquitectónica No 21 (2013). Conversando con...Cruz y Ortiz. Valencia (España): Universitat Politécnica de Valencia.

Vedoya, D. y Prat, E. (2009). Estructuras de Grandes Luces. Tecnología y diseño. Corrientes (Argentina): Ediciones del ITDAHu.

\section{Referencias de Imágenes. Paginas web:}

1. URL: https://www.pgiengineering.com/proyectos/estadio-de-la-peineta

2. URL: Link: http://nuevoestadioatleti.blogspot.com/p/nuevo-apartado-en-construccion_16.html

3. URL:https://www.cruzyortiz.com/portfolio/estadio-de-futbol-del-club-atletico-de-madrid/

4. URL: https://elpais.com/ccaa/2017/07/30/madrid/1501430607_769630.html

5. URL: http://nuevoestadioatleti.blogspot.com/p/nuevo-apartado-en-construccion_16.html

6. URL: https://www.cruzyortiz.com/portfolio/estadio-de-futbol-del-club-atletico-de-madrid/

7.-8. URL: http://nuevoestadioatleti.blogspot.com/2016/03/marzo-2016.html

9. URL: https://en.atleticodemadrid.com/galerias/the-evolution-of-the-works-of-the-new-atletico-demadrid-stadium

10. Secciones Capitel: Martínez Calzón, J., Gómez Navarro, M. y Schlaich, M. (2009) El proyecto de la estructura del Estadio Olímpico de Madrid - Madrid (España).

11. URL: https://www.cruzyortiz.com/portfolio/estadio-de-futbol-del-club-atletico-de-madrid/

12. URL: https://www.mc2.es/proyecto/estadio-metropolitano/

13. URL: http://nuevoestadioatleti.blogspot.com/p/caracteristicas.html

14.URL: http://nuevoestadioatleti.blogspot.com/p/nuevo-apartado-en-construccion_16.html-

https://www.cruzyortiz.com/portfolio/estadio-de-futbol-del-club-atletico-de-madrid/

15. URL: https://www.archdaily.com/877598/time-lapse-shows-the-roof-installation-at-madrids-wandametropolitano-stadium/598dd201b22e382ff4000027-time-lapse-shows-the-roof-installation-at-madridswanda-metropolitano-stadium-photo?next_project=no

16. URL: https://www.cruzyortiz.com/portfolio/estadio-de-futbol-del-club-atletico-de-

madrid/?gallery=planos\&start_gallery=13840 y http://nuevoestadioatleti.blogspot.com/p/nuevoapartado-en-construccion_16.html

19. URL: http://nuevoestadioatleti.blogspot.com/p/nuevo-apartado-en-construccion_16.html

20. URL: https://www.atleticodemadrid.com/galerias/comienza-la-instalacion-de-los-paneles-radialesde-la-cubierta?modo=thumbs

21. URL: http://nuevoestadioatleti.blogspot.com/p/nuevo-apartado-en-construccion_16.html

Biela: (Martínez Calzón, J., Gómez Navarro, M. y Schlaich, M.; 2009) 